\title{
A LC-MS/MS CONFIRMATORY METHOD FOR DETERMINATION OF CHLORAMPHENICOL IN REAL SAMPLES SCREENED BY COMPETITIVE IMMUNOASSAY
}

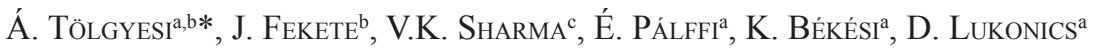 \\ and G. PLEVA ${ }^{\mathrm{a}}$ \\ ${ }^{a}$ National Food Chain Safety Office, Food and Feed Safety Directorate, Food Toxicological National Reference \\ Laboratory, 1095 Budapest, Mester utca 81. Hungary \\ ${ }^{b}$ Department of Inorganic and Analytical Chemistry, Faculty of Chemical and Biotechnology, Budapest University \\ of Technology and Economics, 1111 Budapest, Szt. Gellert tér 4. Hungary \\ 'Department of Environmental and Occupational Health, School of Rural Public Health, Texas A\&M University, \\ 1266 TAMU, College Station, TXT 77843-1266. USA
}

(Received: 29 November 2012; accepted: 9 January 2013)

\begin{abstract}
A new liquid chromatography-tandem mass spectrometric (LC-MS/MS) method was developed to confirm chloramphenicol (CAP) residues in foods of animal origin and in urine samples, which were earlier found positive under the screening analysis, performed by competitive enzyme-linked immunoassay (ELISA) technique. The developed LC-MS/MS method was applied to four non-compliant samples from 2008 to 2012; giving concentrations of CAP residues from 1.18 to $3.68 \mu \mathrm{g} \mathrm{kg}^{-1}$. All samples, qualified positive by ELISA, were confirmed with the LCMS/MS technique and found to be non-compliant. The effectiveness of the confirmatory method was proven by participating in a successful proficiency test in year 2010. Both LC-MS/MS and ELISA methods were validated according to the European Union 2002/657/EC decision. The decision limit of the confirmatory method was determined as $0.02 \mu \mathrm{g} \mathrm{kg}^{-1}$ for CAP in each validated matrix, while the detection capability of the screening test was $0.15 \mu \mathrm{g} \mathrm{kg}^{-1}$.
\end{abstract}

Keywords: chloramphenicol, LC-MS/MS, confirmation, validation, ELISA

Chloramphenicol (CAP) is widely applied as an effective antibiotic drug in veterinary practice. This antibiotic has several serious health concerns even at low doses (AlLEN, 1985). Numerous countries including the United States, the European Union (EU), Japan, and China have strictly banned the use of CAP in food-producing animals. Unfortunately, illegal uses of CAP in livestock have resulted in its detection in honey, tissues, and shrimp samples (SHEN and JANG, 2005; Shen et al., 2006; Lopez et al., 2008; Chen et al., 2011; FernANDEZ-Torres et al., 2011; Y IBAR et al., 2011). In the EU, a concentration of $0.3 \mu \mathrm{g} \mathrm{kg}^{-1}$ has been established as the minimum required performance limit (MRPL) for CAP (EC, 2003). Commonly used microbiological plate test cannot detect such low level of CAP. The competitive enzymelinked immunoassay (ELISA) method has been used successfully in monitoring CAP in samples (Kolosova et al., 2000; PArK et al., 2004; PARK \& Kim, 2006; Shen et al., 2006). However, this method is not suitable for confirmation of CAP in samples of different origins (ImPENS et al., 2003; CRLs, 2010). It is therefore important to develop a highly sensitive analytical method for the detection of CAP in different samples (Bogusz et al., 2004; SHEN and JAnG, 2005; Lopez et al., 2008; Rejtharoví and Rejthar, 2009; Siqueira et al., 2009;

\footnotetext{
* To whom correspondence should be addressed.

Phone: +36-30-96-89-346; fax:+36-1-2161574; email: tolgyesi83@gmail.com
} 
Aresta et al., 2010; Barganska et al., 2011; Berendsen et al., 2011; Chen et al., 2011; Fernandez-Torres et al., 2011; Tian, 2011).

Analytical procedures to determine CAP focus on gas chromatography (GC) and liquid chromatography (LC) coupled to mass spectrometric (MS) detector (IMPENS et al., 2003; Aresta et al., 2010; Berendsen et al., 2011; Chen et al., 2011; Fernandez-Torres et al., 2011). In LC-MS practice, it is very important to evaluate the method performance in incurred samples. Most of the procedures presented in the literature could be applied only to one or two incurred samples (SHen and JiAnG, 2005; SHEN et al., 2006; Chen et al., 2011; Lopez et al., 2008; FernandeZ-Torres et al., 2011; Yibar et al., 2011). In the present paper, an LC-MS/MS method was developed for chicken meat, liver, bovine milk, and porcine urine matrices. Significantly, the newly developed method confirmed chloramphenicol residue in various incurred samples and was also successful in proficiency test sampling.

\section{Materials and methods}

\subsection{Reagents, equipments, and instruments}

HPLC grade methanol and ethyl acetate were obtained from Promochem (Wesel, Germany). Suprapur acetic acid (100\%) was purchased from Merck (Budapest, Hungary). Analytical standards, as chloramphenicol (99.8\%) and chloramphenicol-d5 deuterium labelled internal standard (97\%), were purchased from Sigma-Aldrich (Budapest, Hungary). Ammonium acetate of $99.999 \%$ was obtained from Sigma-Aldrich (Budapest, Hungary). Stock solution of chloramphenicol $\left(1 \mathrm{mg} \mathrm{m}^{-1}\right)$ was prepared by dissolving $10.0 \mathrm{mg}$ standard in $10 \mathrm{ml}$ of methanol. This solution was stored at $-20^{\circ} \mathrm{C}$ for a month. The working standard solution was prepared daily in water by diluting the stock solution. The blank and fortified samples for method development and validation originated from the Hungarian residue control monitoring program from January 2008 to July 2012 and were stored at $-20^{\circ} \mathrm{C}$ until subjected to analysis.

Phenomenex Strata-XL SPE cartridges, Gemini C-18 HPLC column, and Gemini C-18 guard column were purchased from Gen-lab Ltd. (Budapest, Hungary). The ELISA instrument was Thermo Multiskan Ascent (Unicam Ltd., Budapest, Hungary). Elisa test kit for CAP analysis was obtained from R-Biopharm (Budapest, Hungary). The LC-MS/MS separation was carried out by Agilent 6410A Triple Quad LC/MS (Agilent Technologies, Palo Alto, CA, USA) equipped with Agilent 1200 binary pump LC and Agilent 6410A mass selective detector, and an Agilent multimode ion source. Data analysis was performed using Agilent Mass Hunter B 01.04. software.

\subsection{Sample extraction and hydrolyses}

Meat and liver samples (5.0 g) were weighed into $50 \mathrm{ml}$ polypropylene (PP) centrifuge tubes. Samples were extracted with $7 \mathrm{ml}$ ethyl acetate by vortex-mixing for $30 \mathrm{~s}$, followed by shaking on a Janke \& Kunkel IKA KS125 shaker (Staufen, Germany) at $700 \mathrm{~min}^{-1}$ for $20 \mathrm{~min}$ at ambient temperature. Samples were then centrifuged at 10000 r.p.m. with a Sigma 3-18K centrifuge (Osterode am Harz, Germany) at $15^{\circ} \mathrm{C}$ for $10 \mathrm{~min}$. Supernatants were transferred into glass tubes and the extraction was repeated. The upper layers of the two extractions were combined in glass tubes and $2 \mathrm{ml}$ water containing $5 \%$ acetic acid $(\mathrm{v} / \mathrm{v})$ was added to the samples. The samples were vortex-mixed and evaporated to $\sim 2 \mathrm{ml}$ in a TurboVap LV (Hopkinton, MA, USA) under a gentle nitrogen stream at $45^{\circ} \mathrm{C}$. The samples were then 
cooled down to $30-35^{\circ} \mathrm{C}$ at ambient temperature. Four millilitres of distilled water containing $5 \%$ acetic acid was added to the samples, and then re-dissolved by vortex-mixing for $20 \mathrm{~s}$. The samples were then subjected to solid-phase extraction (SPE) clean-up.

For milk samples, $5.0 \mathrm{~g}$ samples were weighed into $50 \mathrm{ml}$ PP tubes. Proteins were precipitated by adding $1 \mathrm{ml}$ acetic acid to the samples, followed by vortex-mixing for $20 \mathrm{~s}$. Samples were centrifuged at 10000 r.p.m. for $10 \mathrm{~min}$ at $15^{\circ} \mathrm{C}$, and the upper layers were purified directly with SPE.

In the case of urine samples, $5.0 \mathrm{ml}$ were weighed into $50 \mathrm{ml} \mathrm{PP}$ tubes, the $\mathrm{pH}$ of the samples was adjusted to $5.2 \pm 0.2$ with acetic acid. Five millilitres of $2 \mathrm{M}$ sodium acetate buffer (pH 5.2) and $20 \mu 1$ of 1 MU Helix Pomatia $\beta$-glucuronidase (Calbiochem) were added to the samples. The samples were vortex-mixed and hydrolysed overnight at $37{ }^{\circ} \mathrm{C}$. The hydrolyzed samples were extracted first with ethyl acetate and the extract was further cleanedup with SPE. The same procedure was followed as for tissue samples.

\subsection{Solid-phase extraction clean-up}

All samples were cleaned-up and concentrated on polymeric Strata-XL (6 ml, $200 \mathrm{mg}$, and $100 \mu \mathrm{m})$ SPE cartridges that were previously conditioned two times with $6 \mathrm{ml}$ methanol, two times with $6 \mathrm{ml}$ water, and finally with $3 \mathrm{ml}$ distilled water containing 5\% acetic acid (v/v). The samples were passed through the cartridges dropwise $\left(\sim 0.3 \mathrm{ml} \mathrm{min}{ }^{-1}\right)$, then the SPE columns were rinsed two times with $6 \mathrm{ml}$ water. The cartridges were dried under vacuum for $60 \mathrm{~s}$ before eluting the samples with $5 \mathrm{ml}$ methanol into glass receiving tubes containing CAP-d5 internal standard (ISTD). The samples were evaporated under a gentle nitrogen stream to dryness at $45^{\circ} \mathrm{C}$. The samples were re-dissolved in $250 \mu 1$ mobile phase by vortexmixing for $20 \mathrm{~s}$, and filtered through $0.45 \mu \mathrm{m}$ Phenex nylon filters (Gen-lab Ltd., Budapest, Hungary) into HPLC vials.

\subsection{Preparation of matrix-matched calibration and spiked samples}

Matrix-spiked calibration of samples was carried out by fortifying blank samples prior to the sample preparation procedure. The calibration solutions for CAP were blank, $0.15 \mu \mathrm{g} \mathrm{kg}^{-1}$, $0.30 \mu \mathrm{g} \mathrm{kg}^{-1}, 0.45 \mu \mathrm{g} \mathrm{kg}^{-1}, 0.60 \mu \mathrm{g} \mathrm{kg}^{-1}$, and $1.2 \mu \mathrm{g} \mathrm{kg}^{-1}$. Internal standard (CAP-d5) was added to the samples at the end of clean-up, and the concentration of ISTD was $0.30 \mu \mathrm{g} \mathrm{kg}^{-1}$ in all samples. Spiked samples were prepared using the same procedure as for the samples of calibration. The accuracy was calculated from the matrix-matched calibration. Incurred samples were also evaluated using the matrix-spiked approach.

\subsection{LC-MS/MS analysis}

Chloramphenicol was separated on Gemini C-18 column $(150 \mathrm{~mm} \times 4.6 \mathrm{~mm}, 5 \mu \mathrm{m})$ equipped with a guard column $(4 \times 3.0 \mathrm{~mm})$ using a linear gradient elution. In the gradient program two solvents (A and B) were mixed. The mobile phase $A$ had $5 \mathrm{mM}$ ammonium acetate and $0.05 \%$ $(\mathrm{v} / \mathrm{v})$ acetic acid in water $(\mathrm{pH} 4.1)$, while the mobile phase $\mathrm{B}$ was $100 \%$ methanol. The gradient elution started from $10 \%$ B that increased to $90 \%$ over $10 \mathrm{~min}$. Then $90 \%$ B was held for $4 \mathrm{~min}$. After $14 \mathrm{~min}$, mobile phase B decreased to $10 \%$ over $0.5 \mathrm{~min}$. The flow rate was $0.8 \mathrm{ml} \mathrm{min}-1$ and the total analysis time was $20 \mathrm{~min}$. The injection volume was $10 \mu \mathrm{l}$ and the thermostat of the analytical column was set at $27^{\circ} \mathrm{C}$. 
The MS/MS detector was set into MRM (multiple reaction monitoring) mode. Two ion transitions (quantifier and qualifier) were applied for CAP, while the internal standard, CAP-d5, was detected with one ion trace. The quantifier ion transition of CAP was $321.0>152.0 \mathrm{~m} / \mathrm{z}$ (fragmentor voltage: $100 \mathrm{~V}$, collision energy: $15 \mathrm{~V}$ ), the qualifier ion transition was $321.0>256.8 \mathrm{~m} / \mathrm{z}$ (fragmentor voltage: $100 \mathrm{~V}$, collision energy: $30 \mathrm{~V}$ ). CAP-d5 was quantified with $326.1>157.1 \mathrm{~m} / \mathrm{z}$ (fragmentor voltage: $100 \mathrm{~V}$, collision energy: $15 \mathrm{~V}$ ) ion trace. Dwell time of $100 \mathrm{~ms}$ and delta electron multiplier voltage of $500 \mathrm{~V}$ were used for all ion transitions. The multimode ion source (MMI) was set in the negative APCI mode. The MMI settings were: drying gas temperature: $300{ }^{\circ} \mathrm{C}$, drying gas flow: $51 \mathrm{~min}^{-1}$, vaporizer: $160{ }^{\circ} \mathrm{C}$, nebulizer pressure: $413.7 \mathrm{kPa}(60 \mathrm{psi})$, capillary voltage: $2500 \mathrm{~V}$, capillary current: $4 \mu \mathrm{A}$. Nitrogen was the collision and drying gas. The collision gas pressure was $1.07 \mathrm{~Pa}$.

\section{Results and discussion}

\subsection{Development of LC-MS/MS method}

Recently, we have reported the optimization of mass spectrometric detection using Agilent 6410A tandem mass spectrometer equipped with multimode ion source (MMI) (TöLGYESI et al., 2012a; 2012b). The same approach was used for CAP. Precursor ion was detected only in negative mode as $[\mathrm{M}-\mathrm{H}]^{-}$. Mother ion was fragmented as written in the above mentioned papers and the ion transitions were checked in MRM mode. The ion traces could be detected with improved sensitivity when MMI was set into APCI (atmospheric pressure chemical ionization) mode. Generally, high vaporizer temperature $\left(>200{ }^{\circ} \mathrm{C}\right)$ results in enhanced responses in APCI mode, however, we found that $160^{\circ} \mathrm{C}$ was the optimal source temperature in MMI. Due to the APCI mode used for ionization, methanol was applied as the organic modifier in the HPLC separation. To force the ionization efficiency, an acetate buffer at $\mathrm{pH} 4.1$ was applied as aqueous mobile phase. At this $\mathrm{pH}$ the ionization of CAP in negative mode could be achieved. Methanol-acetate buffer eluent composition in linear gradient elution mode resulted in sensitive determination of CAP on fully porous Gemini C-18 HPLC column.

For sample preparation, liquid-liquid or solid-liquid extraction using ethyl acetate as extracting solvent is well usable for CAP (YIBAR et al., 2011). However, single extraction is not enough to reduce the matrix effect of LC-MS/MS analysis, and hence further clean-up was necessary. Generally, reversed-phase solid-phase extraction (RP SPE) cleaning and concentration are applied for CAP (BERENDSEN et al., 2013). Polymer based RP SPE cartridge as HLB (hydrophilic lipophilic balance) enables good retention for neutral and medium polar compounds as CAP (BERENDSEN et al., 2013). In this study we used a similar cartridge to HLB, namely Strata-XL. The surface of this SPE column contains both divinylbenze and $\mathrm{N}$-vinylpyrrolidone monomer groups corresponding to non-polar and polar phases, respectively. Since CAP contains both non-polar and polar functional groups, the cartridge can well-retain the CAP on the reversed-phase via hydrophobic interaction. After the sample extraction with ethyl acetate the samples were loaded onto the conditioned cartridges from acidic aqueous solvent. Acid can enhance the dissolution of CAP in the aqueous phase, so 5\% (v/v) acetic acid solution was found to be good for sample re-dissolution after evaporating the ethyl acetate. However, $100 \%$ aqueous solvent is weak enough not to elute CAP from the cartridge, therefore the adsorption of CAP was not limited in this sample solvent composition. 
For rinsing the SPE columns we only applied $100 \%$ water not to start the elution of CAP. For sample elution organic solvents as ethyl acetate, methanol, or acetonitrile can be used. We applied methanol and found that $5 \mathrm{ml}$ is enough to fully elute the CAP from the reversedphase. A deuterium labelled internal standard, CAP-d5 was added to the sample at the end of the preparation to calibrate the ion source response, and consequently, to compensate the matrix effect.

\subsection{Validation of LC-MS/MS method}

The method was validated in accordance with the EU Decision (EC, 2002) using a matrixcomprehensive in-house validation strategy and InterVal software (UhLig et al., 2003; TöLGYESI et al., 2012a; 2012b). Validation results are summarized in Table 1. Meat and liver samples were simultaneously validated. Milk was validated along with urine matrix. In the validation plan, created by InterVal software, one matrix was investigated one day and the other matrix was measured another day (TölgYeSI et al., 2012a; 2012b). Consequently, differences in sample clean-up between matrices did not cause problem. Selectivity of separation was tested by analysing blank and fortified samples. No interfering matrices were detected in different blank samples where CAP eluted from HPLC column, therefore, the requirement of selectivity was proved. The identification was based on the comparison of ion ratios, which is the intensity ratio of qualification and quantification ion transition, in standard solution and spiked samples. The average of ion ratios obtained in standard solution was $27 \%$. The maximum tolerance in samples is $\pm 25 \%$ (EC, 2002), therefore, the permitted range is $20.25-33.75 \%$. Calculated ion ratios in spiked and incurred samples varied between $25.8 \%$ and $30.1 \%$. Linearity was tested between blank to $1.2 \mu \mathrm{g} \mathrm{kg}^{-1}$ for each matrix and determination coefficients $\left(\mathrm{r}^{2}\right)$ were in the range $0.985-0.997$. The fortification levels for CAP were MRPL $\left(0.3 \mu \mathrm{g} \mathrm{kg}^{-1}\right), 1.5 \mathrm{MRPL}\left(0.45 \mu \mathrm{g} \mathrm{kg}^{-1}\right)$, and $2 \mathrm{MRPL}\left(0.6 \mu \mathrm{g} \mathrm{kg}^{-1}\right)$. Eight parallel samples were analysed at each level, and therefore, twenty-four samples were investigated per matrix. Two factors were set. The leading (first) factor was the matrix, the second factor was the operators. Both factors had two levels. Decision limit was determined along with limit of detection (LOD) as three times of signal-to-noise ratio and found to be $0.02 \mu \mathrm{g} \mathrm{kg}^{-1}$ in all matrices (Table 1). Limit of quantification (LOQ) was calculated as $3.33 \times$ LOD. The decision limit and LOD were confirmed by analysing six blank samples that were spiked to $0.02 \mu \mathrm{g}$ $\mathrm{kg}^{-1}$ concentration levels before the sample preparation (Fig. 1). Analytical limits were checked for all matrices, and accepted when the signal-to-noise ratio of both ion transitions were higher than three and the ion ratios were in the permitted tolerance range set by the EU (EC, 2002). The stability tests in both raw and cleaned-up samples are in progress.

Table 1. Validation results using LC-MS/MS technique

\begin{tabular}{|c|c|c|c|c|c|c|}
\hline \multirow{2}{*}{ Matrix } & \multicolumn{3}{|c|}{ Recovery\% (RSD\%) } & \multirow{2}{*}{$\begin{array}{c}\mathrm{CCa} \\
\left(\mu \mathrm{g} \mathrm{kg}^{-1}\right)\end{array}$} & \multirow{2}{*}{$\begin{array}{c}\text { LOD } \\
\left(\mu \mathrm{g} \mathrm{kg}^{-1}\right)\end{array}$} & \multirow{2}{*}{$\begin{array}{c}\mathrm{LOQ} \\
\left(\mu \mathrm{kg}^{-1}\right)\end{array}$} \\
\hline & 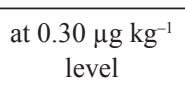 & $\begin{array}{c}\text { at } 0.45 \mu \mathrm{g} \mathrm{kg}^{-1} \\
\text { level }\end{array}$ & $\begin{array}{c}\text { at } 0.60 \\
\mu \mathrm{g} \mathrm{kg}^{-1} \text { level }\end{array}$ & & & \\
\hline Meat & $91.3(27.7)$ & $86.7(23.2)$ & $98.1(17.0)$ & 0.02 & 0.02 & 0.07 \\
\hline Liver & $91.3(27.7)$ & $86.7(23.2)$ & $98.1(17.0)$ & 0.02 & 0.02 & 0.07 \\
\hline Milk & $95.2(11.5)$ & $95.8(16.7)$ & $99.6(14.4)$ & 0.02 & 0.02 & 0.07 \\
\hline Urine & $95.2(11.5)$ & $95.8(16.7)$ & $99.6(14.4)$ & 0.02 & 0.02 & 0.07 \\
\hline
\end{tabular}



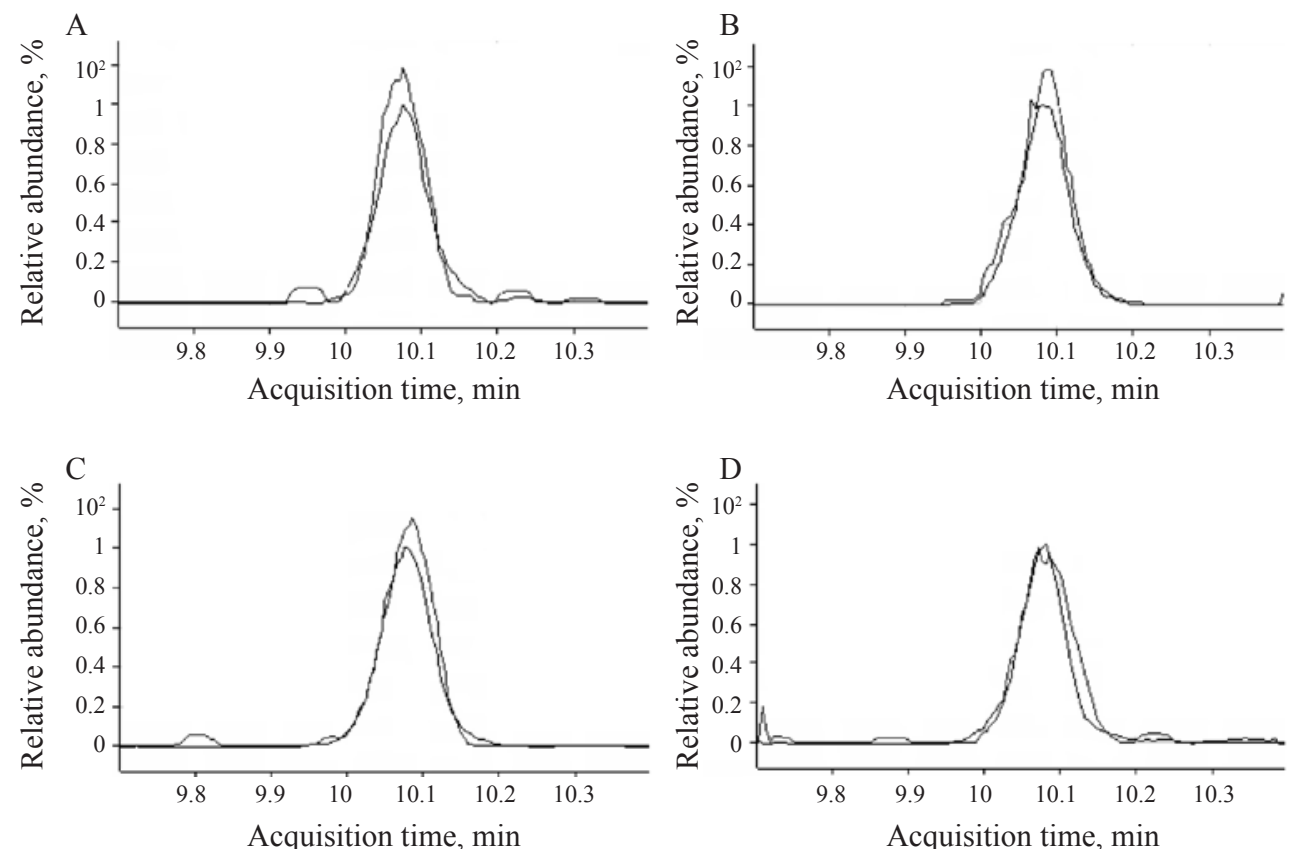

Fig. 1. MRM chromatograms of spiked samples (A: meat; B: liver; C: milk and D: urine) containing $0.02 \mu \mathrm{g} \mathrm{kg}-1$ chloramphenicol

\subsection{Validation of ELISA method}

During the ELISA analysis, the sample preparation and subsequent instrumental determination of food as well as urine samples were made according to R-Biopharm R1505 guideline and its test kit (R-Biopharm RIDASCREEN). R-Biopharm offers another guideline and test kit, R1503 for urine samples, which detected several false positive urine samples in our laboratory. ELISA method was validated for meat, liver, milk, and urine matrices according to guidelines for the validation of screening methods (CRLs, 2010). In the case of each matrix, all of the twenty blank samples, originated from different batches, were analysed on different days. These blank samples were fortified to $0.15 \mu \mathrm{g} \mathrm{kg}^{-1}$ ( $\left.0.5 \mathrm{MRPL}\right)$ and were also analysed. These spiked samples were not measured with LC-MS/MS. The highest response of blank samples was lower than the lowest response of spiked samples, therefore, the detection capability (CC $\beta$ ) of the ELISA method could be established as $0.15 \mu \mathrm{g} \mathrm{kg}^{-1}$ (Table 2). The Cut-Off level in different matrices (Table 2) was determined from the lowest response (absorbance) of spiked samples (Community Reference Laboratories (CRLs) 20/1/2010). Those samples that add lower responses than the Cut-Off level can be qualified as positive.

\subsection{Confirmation of real samples}

From 2008 to 2012, four samples (one chicken meat and liver, one bovine milk, and one porcine urine) were qualified as being positive for CAP during the screening measurements. All of these samples were confirmed by LC-MS/MS and also found to be non-compliant. The ELISA method has not given false positive results yet. It should be pointed out that the aim 
Table 2. Validation results using ELISA technique

\begin{tabular}{lcc}
\hline Matrix & $\begin{array}{c}\mathrm{CC} \beta \\
\left(\mu \mathrm{g} \mathrm{kg}^{-1}\right)\end{array}$ & $\begin{array}{c}\text { Cut-Off } \\
(\text { Absorbance })\end{array}$ \\
\hline Meat & 0.15 & 1.048 \\
Liver & 0.15 & 0.790 \\
Milk & 0.15 & 0.493 \\
Urine & 0.15 & 0.573 \\
\hline
\end{tabular}

of a screening method is to find the questionable samples and not to give final results on them. In case of meat and liver samples 0.35 and $0.82 \mu \mathrm{g} \mathrm{kg}^{-1} \mathrm{CAP}$ were detected, respectively, using ELISA. Confirming the meat and liver samples by LC-MS/MS method, 1.18 and $1.66 \mu \mathrm{g} \mathrm{kg}^{-1} \mathrm{CAP}$ were detected, respectively. In the milk sample $0.34 \mu \mathrm{g} \mathrm{kg}^{-1} \mathrm{CAP}$ was found during the screening test, while the confirmatory method added $1.19 \mu \mathrm{g} \mathrm{kg}^{-1}$ for CAP. For urine sample $0.84 \mu \mathrm{g} \mathrm{kg}^{-1}$ CAP could be detected by ELISA, while the LC-MS/MS method gave a result of $3.68 \mu \mathrm{g} \mathrm{kg}{ }^{-1}$ for CAP. The ratios of concentrations detected with different techniques depended on the matrix of interest.

In 2010 a proficiency test called "Analysis of Chloramphenicol Residues in Aquaculture Products" was organized by ANSES EU-RL (Fougeres, France). Three trout samples had to be analysed for CAP using the confirmatory method. We measured the samples with both screening and confirmatory methods. The absorbance of a blank trout sample was 1.856 unit during ELISA analysis. From the three proficiency test samples, one sample had the same response (1.844 unit) and was qualified as being negative by ELISA. Other two samples were found to be positive giving 1.464 and 1.351 absorbances, respectively. All samples were confirmed with the described LC-MS/MS method. In the negative sample CAP was not detected, while in the positive samples $0.15 \mu \mathrm{g} \mathrm{kg}^{-1}$ and $0.23 \mu \mathrm{g} \mathrm{kg}^{-1}$ of CAP were found. ELISA could well screen the CAP again. According to the final report of the proficiency test, the positive samples contained $0.15 \mu \mathrm{g} \mathrm{kg}^{-1}$ and $0.21 \mu \mathrm{g} \mathrm{kg}^{-1}$ of CAP. The calculated $z$ scores were -0.28 and -0.37 . The proficiency test is successful if the $z$ scores are between -2 and 2 . These results showed that the method was successful in this performance test.

\section{Conclusions}

A new LC-MS/MS method was developed to confirm CAP residues in food matrices and in urine samples. The screening analysis, performed by ELISA test, resulted in no false positive values. The ELISA test and subsequent LC-MS/MS confirmation could be used for the detection of CAP in incurred meat liver, milk, and urine samples. Both methods were successfully validated for these matrices according to the EU standards. The performance of the LC-MS/MS method was demonstrated by participating in a successful proficiency test.

We wish to thank P. IMrIK and T. SZÁsz VADÁsz (Gen-lab Ltd) who provided the equipments for the measurements. We wish to acknowledge the support of G. DománY (Head of Laboratory, Food Toxicological NRL). 


\section{References}

Allen, E.H. (1985): Review of chromatographic methods for chloramphenicol residues in milk, eggs, and tissues from foodproducing animals. J. Assoc. Anal. Chem., 68, 990-999.

Aresta, A., Bianchi, D., Calvano, C.D. \& Zambonin, C.G. (2010): Solid phase microextraction-liquid chromatography (SPME-LC) determination of chloramphenicol in urine and environmental water samples. J. Pharm. Biomed. Anal., 53, 440-444.

Barganska, Z., Slebioda, M. \& Namiesnik, J. (2011): Determination of antibiotic residues in honey. TrAC-Trend Anal. Chem., 30, 1035-1041.

Berendsen, B.J.A., Essers, M.L., Stolker, A.A.M. \& Nielen, M.W.F. (2011): Quantitative trace analysis of eight chloramphenicol isomers in urine by chiral liquid chromatography coupled to tandem mass spectrometry. $J$. Chromatogr. A., 1218, 7331-7340.

Berendsen, B.J.A., Stolker, A.A.M. \& Nielen, M.W.F. (2013): Selectivity in the sample preparation for the analysis of drug residues in products of animal origin using LC-MS. TrAC-Trend Anal. Chem, 43, 229-239.

Bogusz, M.J., Hassan, H., Al-EnAZi, E., IBrahim, Z. \& Al-Tufail, M. (2004): Rapid determination of chloramphenicol and its glucuronide in food products by liquid chromatography-electrospray negative ionization tandem mass spectrometry. J. Chromatogr. B, 807, 343-356.

Bononi, M. \& TATEO, F. (2008): Liquid chromatography/tandem mass spectrometry analysis of chloramphenicol in propolis extracts available on the Italian market. J. Fd Compos. Anal., 21, 84-89.

Chen, X.B., Wu, Y.L. \& YAng, T. (2011): Simultaneous determination of clenbuterol, chloramphenicol and diethylstilbestrol in bovine milk by isotope dilution ultraperformance liquid chromatography-tandem mass spectrometry. J. Chromatogr. B., 879, 799-803.

EC (2002): Commission Decision 2002/657/EC., Off. J. EU Comm., L221/8.

EC (2003): Commission Decision 2003/181/EC., Off. J. EU Comm., L71/17.

CRLs (2010): Guidelines for the validation of screening methods for residues of veterinary medicines (Initial validation and transfer). Community Reference Laboratories 20/1/2010. http://ec.europa.eu/food/food/ chemicalsafety/residues/Guideline_Validation_Screening_en.pdf

Fernandez-Torres, R., Bello lopez, M.A., Olias Consentino, M., Callejon Mochon, M. \& Ramos Payan, M. (2011): Enzymatic-microwave assisted extraction and high-performance liquid chromatography-mass spectrometry for the determination of selected veterinary antibiotics in fish and mussel samples. J. Pharm. Biomed. Anal., 54, 1146-1156.

Impens, S., Reybroeck, W., Vercammen, J., Courtheyn, D., Ooghe, S., Wasch, K.D., Smedts, W. \& De Brabander, H. (2003): Screening and confirmation of chloramphenicol in shrimp tissue using ELISA in combination with GC-MS² and LC-MS². Anal. Chim. Acta, 483, 153-163.

Kolosova, A.Y., Samsonova, J.V. \& Egorov, A.M. (2000): Competitive ELISA of chloramphenicol: Influence of immunoreagent structure and application of the method for the inspection of food of animal origin. Fd Agric. Immun., 12, 115-125.

Lopez, M.I., Pettis, J.S., Smith, I.B. \& Chu, P.S. (2008): Multiclass determination and confirmation of antibiotic residues in honey using LC-MS/MS. J. Agric. Fd Chem., 56, 1553-1559.

PARK, I.S., KIM, D.K. \& KIM, N. (2004): Responses of chloramphenicol immunosensor to analyte types. J. Microbiol. Biotech., 14, 1157-1162.

PARK, I.S. \& KIM, N. (2006): Development of a chemiluminescent immunosensor for chloramphenicol. Anal. Chim. Acta, 578, 19-24.

Rejtharová, M. \& Rejthar, L. (2009): Determination of chloramphenicol in urine, feed water, milk and honey samples using molecular imprinted polymer clean-up. J. Chromatogr. A, 1216, 8246-8253.

SHEN, H.Y. \& JiANG, H.L. (2005): Screening determination and confirmation of chloramphenicol in seafood, meat and honey using ELISA, HPLC-UVD, GC-ECD, GC-MS-EI-SIM and GCMS-NCI-SIM methods. Anal. Chim. Acta, 535, 33-41.

Shen, J., Zhang, Z., Yao, Y., Shi, W., Liu, Y. \& Zhang, S. (2006): A monoclonal antibody-based time-resolved fluoroimmunoassay for chloramphenicol in shrimp and chicken muscle. Anal. Chim. Acta, 575, 262-266.

Siqueira, S.R.R., Donato, J.L., Nucci, G. \& Reyes, F.G.R. (2009): A high-throughput method for determining chloramphenicol residues in poultry, egg, shrimp, fish, swine and bovine using LC-ESI-MS/MS. J. Sep. Sci., 32, 4012-4019.

TIAN, H. (2011): Determination of chloramphenicol, enrofloxacin and 29 pesticides residues in bovine milk by liquid chromatography-tandem mass spectrometry. Chemosphere, 83, 349-355. 
Tölgyesi, Á., Fekete, J., Fekete, S., Sharma, V.K., Békési, K. \& Tóth, E. (2012a): Analysis of sub $\mu$ g/kg lincomycin in honey, muscle, milk, and eggs using fast liquid chromatography-tandem mass spectrometry. J. Chromatogr. Sci., 50, 190-198.

Tölgyesi, Á., Sharma, V.K., Fekete, S., Ferete, J., Simon, A. \& Farkas, S. (2012b): Development of a rapid method for the determination and confirmation of nitroimidazoles in six matrices by fast liquid chromatographytandem mass spectrometry. J. Pharm. Biomed. Anal., 64-65, 40-48.

Uhlig, S., Gowik, P. \& RADEck, W. (2003): Performance of a matrix-comprehensive in-house validation study by means of an especially designed software. Anal. Chim. Acta, 483, 351-362.

Yibar, A., Cetinkaya, F. \& Soyutemiz G.E. (2011): ELISA screening and liquid chromatography-tandem mass spectrometry confirmation of chloramphenicol residues in chicken muscle, and the validation of a confirmatory method by liquid chromatography-tandem mass spectrometry. Poult. Sci., 90, 2619-2626. 